\title{
DESIGN AND DEVELOPMENT OF WIRELESS SENSOR NODES DEPLOYMENT SCHEME FOR DISTRIBUTED WSNS
}

\author{
Janappa R. Hugar ${ }^{1}$, Sanjeevakumar M. Hatture ${ }^{2}$ \\ ${ }^{1}$ Selection Grade Lecturer in Electronics \& Communication Dept. Govt. Polytechnic, Bagalkot, Karnataka, India \\ ${ }^{2}$ Associate Prof., Dept. of Computer Science and Engineering, Basaveshwar Engineering College, Bagalkot, \\ Karnataka, India
}

\begin{abstract}
In distributed sensor networks, randomly distributed sensor nodes have to find out its own positions to scale the records to locations. Because of the scare energy supplies of a distributed WSNs it is required to develop the architecture and working of the network so as to optimize the consumption of energy. It is necessary to minimize the distance travelled by a packet to reach the sink because in Multihop WSN a sensor's maximum energy is spent on replaying of data packets. So as to reduce the distance and delay the packet has to travel, properly position sensors in the network is important. This Paper presents the event driven approach to find the maximum information reception position in the wireless sensor Network by positioning the sensor with less depletion of energy of the sensor nodes. We have developed the sensor-positioning algorithm, which position the sink in the network near to the sensor node, which has maximum information about the event. Sensor positioning algorithm investigates, designs and analyzes sensor-positioning model for maximum information reception, as well consumption of less energy position in the network and hence elongate life of the Wireless Senor Network (WSN).
\end{abstract}

Keywords: Multihop WSN, Distributed WSNs, Energy, Sensor-Position, optimization

\section{INTRODUCTION}

In the absence of installed infrastructure the communication takes place between the wireless hosts of distributed WSNs. They have reliable applications in disaster relief, conference, and military environments, and have got major attention in recent days. There are currently two variations of mobile wireless networks. The first type of network topology is the infrastructured network. In infrastructured topology base station will handover the traffic from one station to another station. The base station regulates the arbitration of radio sources. It is not necessary for the nodes to know the communicating path between them. The source and destination nodes have to be within coverage area of base station. The communication ends if the condition is not fulfilled. This kind of centralized control is widely used in GSM, UMTS etc.

The second type of network topology is the infrastructure less networks. In this network architecture if nodes are not in the range of the network will communicate through the intermediate node, which lies in the network range. This intermediate node is known as router, will forward the packets between the nodes. A router is like a server node which identifies the path through which a packet can be delivered to its end destination. By knowing the current status of network the router selects the next node to which a packet can be sent. The Infrastructure less network concept, which developed nowadays, is Ad-hoc networks. In discovery of nodes regrouping the routers are built dynamically due to the enhanced feature of distributed WSNs. So, distributed WSNs are more sensitive to topology changing than any wired networks. This led to the new concept of networking that is simple; enable devices to form a temporary community without any planned installation, or human intervention, known as Mobile Ad-hoc Networks (MANET). Infrastructure less networks are used to transfer information of one device to another if nodes exist between cellular ranges. In some applications like military field, surveillance, disaster recovery etc., where the planned structure is absent. For such mentioned applications network must be deployed on the fly. The new network technology introduced for such mentioned applications is the Wireless Sensor Network (WSN).

Wireless sensor networks [6] is one of the popular present research technology, due to the numerous applications, and more challenging open issues that are yet to be resolved for these applications to perform reliably and efficiently. They are composed of small, low cost sensors with limited energy processing power and memory.

WSN has got popularity due to the enhanced military and civilian surveillance fields applications. Sending information effectively is a challenging issue in WSNs. Using intermediate nodes messages can be delivered to the sink directly. Information delivery to the one of the sinks in the wireless sensor network is important issue and it makes use of different sensor routing protocols. Above all these is the position of the sink node in the wireless network is very import issue. Energy consumption in WSNs is one of the major issues [6] .It is difficult task to change or recharge the batteries of sensors. Thus, energy should be spared, so as to be able to perform, for more period. Energy is consumed by many tasks, but the highest energy consumer is the 
communication module[9]. Sensor nodes can send data directly to the sink in small networks but multi-hop communication is established in large networks., The consumption of energy depends on the communication distance in both cases.

The sink is one of the sensor nodes with high resources (memory, processing power, energy). Depending on the position of the sink node in wireless sensor network, the amount of information about an event varies. The sink node must be placed in such a position where it receives maximum information. This issue has been addressed in our work.

The remaining section of the paper is organized as follows. Section II describes a review of sink deploying solutions and WSNs with multiple sinks. In order to minimize the distance between sensor node and sink a mathematical model is composed in Section III. In section IV we present sensor-positioning algorithm. The algorithm finds the optimal position for the sink in WSNs to get maximum information with least energy requirement and least time delay of reach of information. Section $V$ introduces the simulation of the algorithm and results and discussions. The conclusion of paper and future research aspects are included in section VI.

\section{RELATED WORK}

The sensor deployment phase is an important aspect of WSNs. However, deploying solutions were mainly designed for sensors so as to ensure coverage and connectivity. Even though sink deployment is also be critical in concern with the consumption of energy and life of network.

The energy consumption in communication can be decreased by reducing the distance between sensor and sink using many sinks in WSNs.

In many proposed papers, the way to find the optimal position of multiple sink nodes is addressed. A common way to resolve the issue is to formulate as integer linear programming (ILP) task. In our proposed system we have considered the event driven approach. The previous works on the same concept have considered the time driven approach [1-5][9-13]for positioning a sink in the wireless sensor network.

\section{MATHEMATICAL MODEL}

In this paper we propose a sink-deploying algorithm[9] that reduces the energy consumption in the network. For packets transmission maximum energy is spent. So we have to understand that how the energy is consumed in the multihop network.

If the packets are transmitted using a shortest path algorithm Then Emessage is given by

$$
\text { Emessage }[d / \mathrm{rc}] \text { Ehop }
$$

Where,

Emessage is the required energy to transmit a packet $d$ is the distance between sensor node and closest sink, $r c$ is the radio communication range .

From the equation we understand that the energy consumption to send a message to the sink is directly proportional to the distance $d$. So in order to limit the energy consumption for communication we need to minimize the sum of distances between sensor nodes and sink. Which is nothing but shortening the average distance of sensors and nearest sink. A mathematical model which works in deploying a single sink. This can be extended for deployment of the multiple sinks.

Single sink

The distance between a given sensor and the sink is given by

$$
d i=\sqrt{\left((x i-x)^{2}+(y i-y)^{2}\right.} \quad i=1, . . \mathrm{N} .
$$

Where,

$\mathrm{N}$ is the no. of nodes

$(\mathrm{x}, \mathrm{y})$ is the position of the sink.

$D i$ is the distance between ith node and sink.

The aim is to reduce the average distance from the sink,

$$
\text { i.e., } \quad \sum_{(i=1, . . \mathrm{N})} d i-----\min .
$$

The optimal coordinates of sink are given by

$$
\begin{gathered}
\left.(x 0, y 0)=\arg \min \overline{\sum \sqrt{\left((x i-x)^{2}+(y i-y)^{2}\right.}}\right) \\
(x, y)(i=1 \ldots \mathrm{N})
\end{gathered}
$$

By making partial derivates equal to zero, we can obtain the minimum value

$$
\begin{gathered}
\partial / \partial \mathrm{x} \sum d i=\partial / \partial \mathrm{y} \sum d i=0 \\
(i=1, \ldots \mathrm{N}) @ x=x 0 \quad(i=1, \ldots \mathrm{N}) @ y=y 0
\end{gathered}
$$

The partial derivatives are

$$
\begin{gathered}
\partial / \partial \mathrm{x} \sum d i=\sum \partial / \partial \mathrm{x} d i=\sum(x-x i) / d i \\
(i=1 \ldots \mathrm{N}) \\
\left.\begin{array}{c}
\left.\partial / \partial \mathrm{y} \sum d i=1 \ldots \mathrm{N}\right) \\
(i=1, \ldots \mathrm{N})
\end{array}\right]=\sum \partial / \partial \mathrm{y} d i=\sum(y-y i) / d i \\
(i=1, \ldots \mathrm{N})
\end{gathered}
$$$$
\mathrm{e} i=\mathrm{d} i / d i=(1 / d i)((x-x i),(y-y i))
$$

where

e $i$ be the unit vector directing from the $i$ th node towards the closest sink. the vector directing to position of the ith node is $\mathrm{n} i=(x i, y i)$, the distance vector between the sensor and the sink is $\mathrm{d} i=\mathrm{n} i-\mathrm{s}=((x i-x),(y i-y))$.

Using (6) and (7), the requirements of (5) are equivalent of having 


$$
\begin{gathered}
\mathrm{r}=\sum \mathrm{e} i \mid \quad=0, \\
i=(1 \ldots \mathrm{N}) \mid \mathrm{s}=(x 0, y 0)
\end{gathered}
$$

i.e. ., If the resultant vector $r$ is zero then average distance is shortened.

This mathematical model can be used for multi sinks with little modifications.

\section{SENOR POSITIONING}

The mathematical model shows that, if the sinks are positioned in such a way that every resultant vector is zero, then the average distance between sensor and nearest sink is shortened. The task of positioning of multiple sinks is same as cluster problem. We have considered a cluster in which positioning a sink to receive maximum information by minimizing time delay of information reach and distance of sink from source.

In this paper we present Sensor Positioning Algorithm for finding the position of the sink in a cluster to receive maximum information and utilizing less energy, less time delay. By this energy of the wireless sensor networks is less depleted and this elongates the life of the wireless Networks. The algorithm considers the event driven approach. When ever an event occurs in the region of interest (ROI) sink moves step by to the optimal position where it receives the maximum information, towards the source and event. Thus finding the optimal position for the sink energy of the sensor nodes of the network is less depleted.

\subsection{Assumed Network Model}

The proposed model assumes an event driven and heavily deployed sensor network where sensor senses the information. The sensor nodes use multi-hop communication, the information travel to the nearest sink using AODV. In order to select end to end path and inform the participating nodes there is no central authority. The nodes hand over data packet to the neighbor nodes, which lie in its radio range. Sensors should know the sink's position in order to send the data. It's done through the shortest path. AODV is one of the most widely used routing protocols. This not only help the network to produce better result but also the updating the existing network would not require any significant changes.

\subsection{Sensor Positioning Algorithm}

The main aim of the proposed system is to read the sink node and determine the optimal position of the sink in WSNs where it gets the maximum information. The maximum information reception is interpreted as the energy required to receive that information and as well the time delay of information reach. According to these requirements the system is designed into loosely coupled six modules. Among these, one module is the main, it calls other remaining modules.

\section{SIMULATION RESULTS}

The results and discussions of the proposed systems are elaborated in the following sections. The results of the developed system are tested by simulator. The simulator is developed, using C++ backend and VB 6.0 front end. The Main menu of the simulator looks as below.

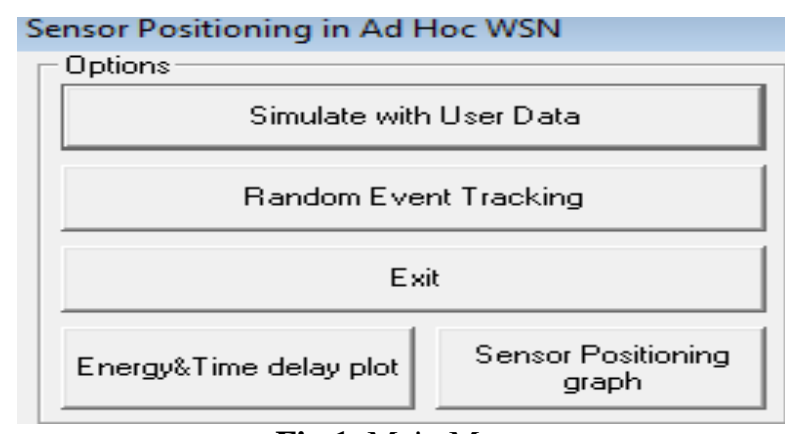

Fig 1. Main Menu

The Figure 1. shows the Main menu of the simulator developed to test the results of the proposed sensor positioning system. The main menu has the following functional units. The first button in the above main menu tests the system for the first case. The second button of the main menu tests the system for the second case and the third button quits the simulator. The fourth button of the main menu displays graph of the energy required to get information and time delay of reach of information.

The proposed system is tested for the following cases using the simulator developed.

- Positioning of the sink in Wireless Senor Ad hoc Network for user given event.

- Positioning of the sink in Wireless Sensor ad hoc Network, for random event.

The following sections describe the above cases with their corresponding results obtained from the simulation of proposed sensor positioning architecture.

\subsection{Positioning of the Sink in Wireless Sensor Ad-}

\section{Hoc Network for user given Event.}

Table 1 Energy of information reach (User Given)

\begin{tabular}{|c|c|}
\hline Number of steps & Energy in $\mathbf{~ J ~}$ \\
\hline 1 & 320.00 \\
\hline 2 & 230.4 \\
\hline 3 & 142.4 \\
\hline 4 & 64 \\
\hline
\end{tabular}

In this case the proposed Sensor Positioning architecture is tested for user given event topology as shown in figure 2 Initially sensor nodes are deployed randomly in the Region of Interest .We are deploying fifteen number of sensor nodes in the ROI for clarity of the movements of the sensors. One of the sensor nodes is selected as the sink node. The nodes are organized in their radio range. User is opted to select the position of event in the ROI. The node which is closer to the event is selected, which finds the routing path to sink 
through the adjacent sensor nodes using AODV protocol and this node is known as source. The average information is routed through the path formed by AODV protocol. Sink knowing the position information of the source and event, it moves step by step towards the radial area of source. This case is simulated to find the energy required for sink to receive the information and as well the time delay of information reach. The values of energy and time delay are tabulated and plotted on graph. The energy required to receive information goes on decreasing as the sink moves towards source and as well the time delay.
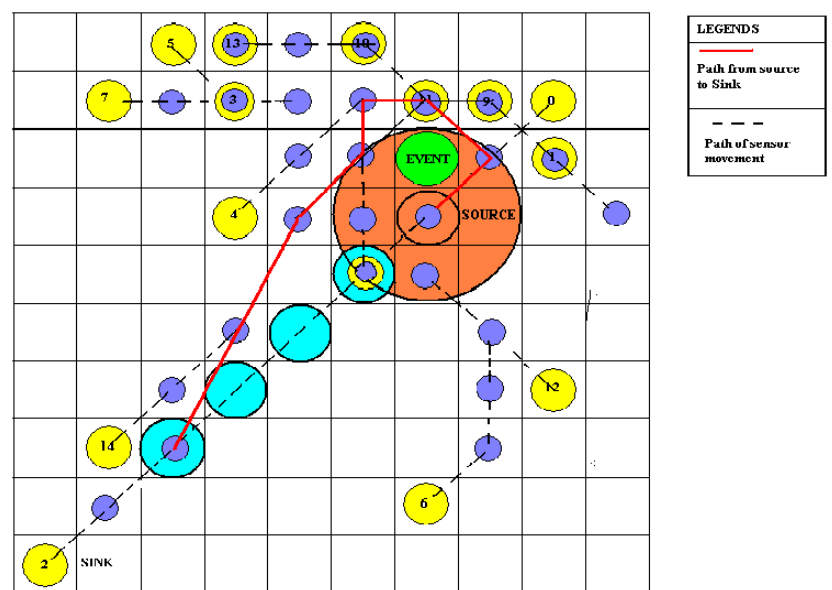

Fig 2. User given event System topology

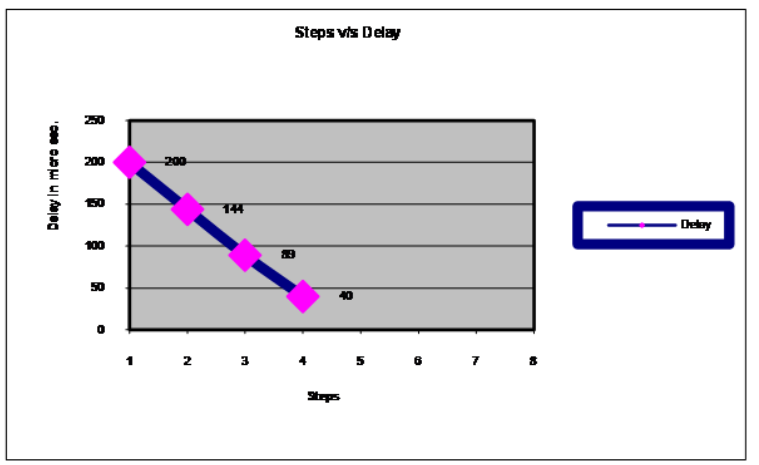

Fig 3 Plot of Number of Steps v/s. Energy

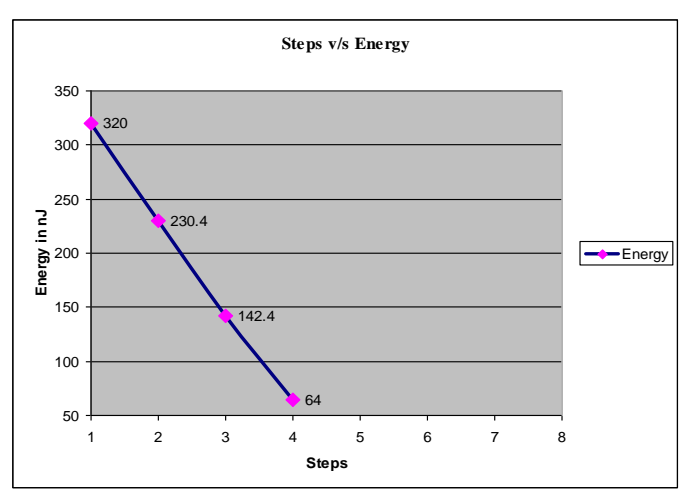

Fig 4 Plot of Number of Steps v/s. Time delay

Table 2 Time delay of information reach (User Given Event)

\begin{tabular}{|c|c|}
\hline Number of steps & $\begin{array}{c}\text { Time delay in } \\
\text { micro sec. }\end{array}$ \\
\hline
\end{tabular}

\begin{tabular}{|c|c|}
\hline 1 & 200 \\
\hline 2 & 144 \\
\hline 3 & 89 \\
\hline 4 & 40 \\
\hline
\end{tabular}

Table 3 Energy of information reach (Random Event)

\begin{tabular}{|c|c|}
\hline Number of steps & Energy in nJ \\
\hline 1 & 256 \\
\hline 2 & 192 \\
\hline 3 & 128 \\
\hline 4 & 64 \\
\hline 5 & 0 \\
\hline
\end{tabular}

\subsection{Positioning of the Sink in Wireless Sensor Ad}

\section{Hoc Network for Random Event}

In this case the proposed Sensor Positioning architecture is tested for random event topology as shown in fig 5 initially sensor nodes are deployed randomly in the Region of Interest. We are deploying fifteen number of nodes in the ROI. One of the nodes is selected as the sink node. The nodes are organized in their radio range. The event is randomly generated in the ROI. The node, which is closer to the event, is selected, which finds the routing path to sink through the adjacent sensor nodes using AODV protocol and this node is known as source. The average information is routed through the path formed by AODV protocol. Sink knowing the position information of the source and the event, it moves step by step towards the source.

This case is simulated to find the energy required for the sink to receive the information and as well the time delay of information reach. The values of energy and time delay are tabulated and plotted on graph. The energy required to receive information goes on decreasing as the sink moves towards source and as well the time delay.

\section{DISCUSSION}

We have tested the system for the two of the cases as elaborated in the previous section.

\subsection{Case 1: Positioning of the Sink in Wireless}

\section{Sensor Ad Hoc Network for User Given Event}

In this case Node 2 is selected as the Sink node. Position of the event given by the user is $26^{\text {th }}$ cell in the region of interest (ROI) as shown in figure 2. The node, which is closer to this position of the event, is Node 0 . This Node 0 is known as Source. The numbered circles are the original positions of the sensor nodes. After deployment sensor nodes move two steps to get the position information and the new positions of the nodes are shown by the small circles. 


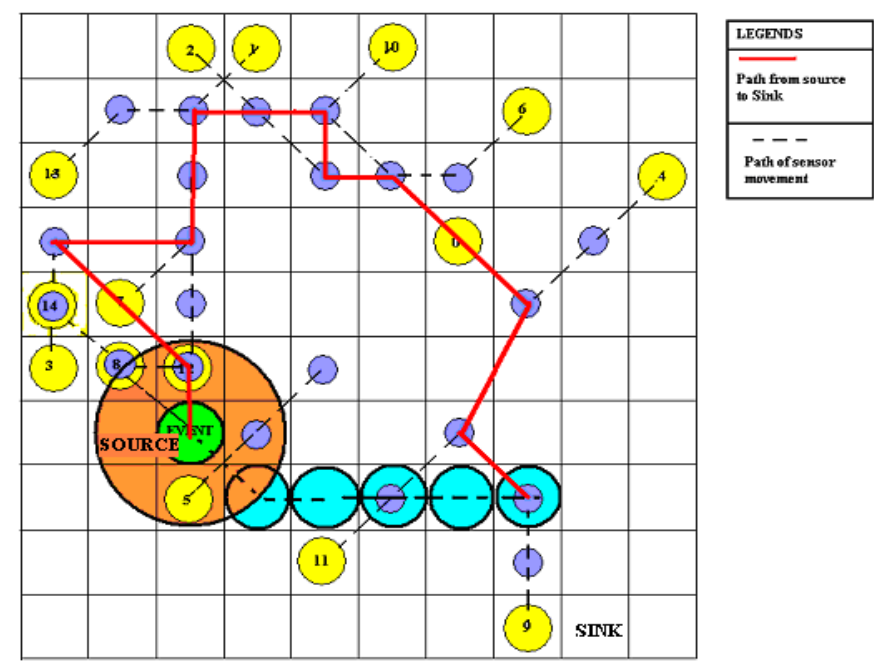

Fig 5 Random event System topology (Random Event)

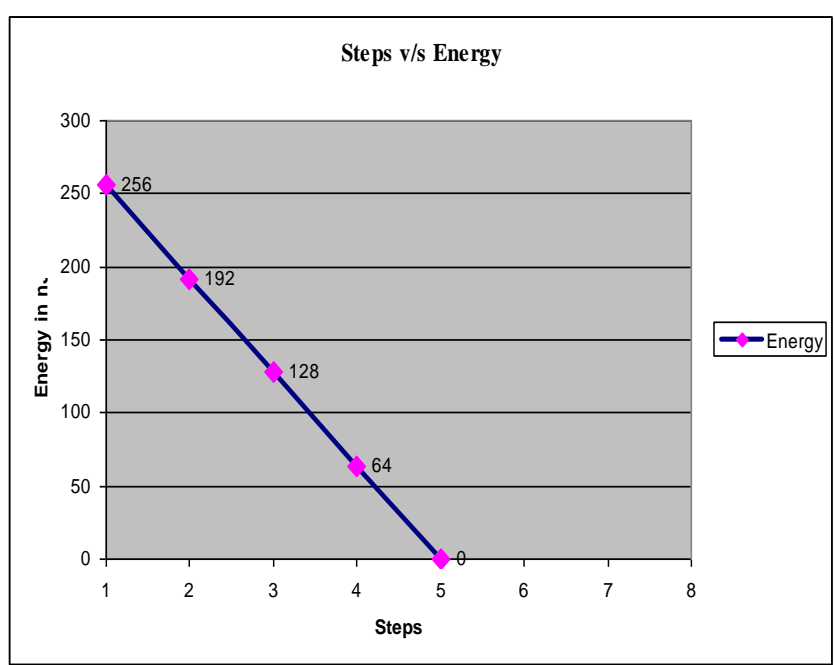

Fig 6 Plot of Number of Steps v/s. Energy

\subsection{Case 2: Positioning of the Sink in Wireless}

\section{Sensor Ad Hoc Network for Random Event.}

In this case Node 9 is selected as the Sink node. Position of the event is randomly generated in the region of interest (ROI) as shown in figure 5. The node, which is closer to this position of the event, is Node 14. This Node14 is known as Source. The numbered circles are the original positions of the sensor nodes. After deployment sensor nodes move two steps to get position information and the new positions of the nodes are shown by the small circles. In the next step the shortest path is found from source to sink and through this path the information flows to sink. Getting the information of the event the sink moves towards the source step by step to get maximum information. The energy of the information and time delay of reach of information are calculated and tabulated. The result is shown in the figures $2,3,4$, table 1 and table2.
Table 4 Time delay of information reach(Random Event)

\begin{tabular}{|c|c|}
\hline Number of steps & $\begin{array}{c}\text { Time delay in micro } \\
\text { sec. }\end{array}$ \\
\hline 1 & 160 \\
\hline 2 & 120 \\
\hline 3 & 80 \\
\hline 4 & 40 \\
\hline 5 & 0 \\
\hline
\end{tabular}

In the next step the shortest path is found from source to sink and through this path the information flows to sink. Getting the information of the event the sink moves towards the source step by step to get maximum information. The energy of the information and time delay of reach of information are calculated and tabulated. The result is shown in the figures $5,6,7$, table 3 and table 4 .The sensor positioning graph represent actual energy, position \& predicted energy, position of sensor nodes

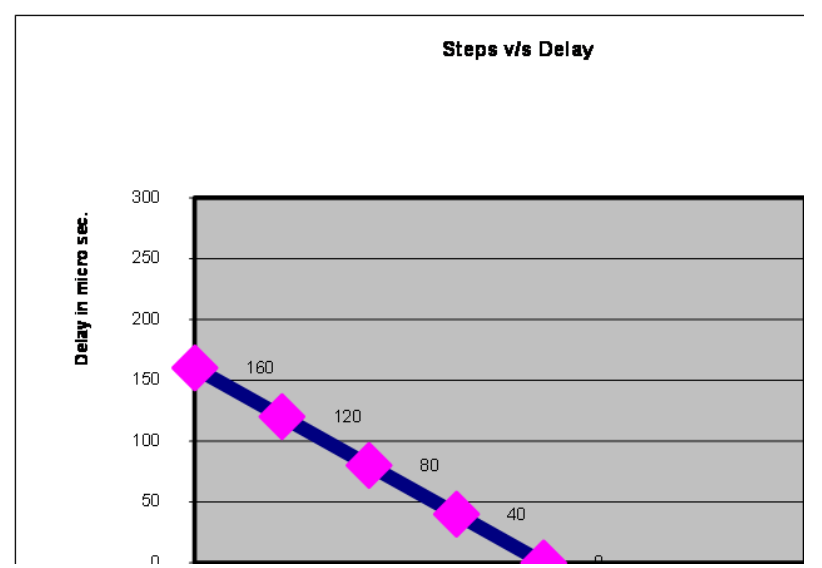

Fig 7 Plot of Number of Steps v/s. Time delay

\section{CONCLUSION AND FUTURE WORK}

The proposed system for positioning a sink in wireless sensor ad-hoc network for maximum information reception. Mathematical model for the system that helps in real time to position the nodes in the sensor wireless network where human intervention is not possible. The developed system also elongates the life of the senor network by minimizing the energy required to receive the information and as well the time delay of reception. The works related to this area and problem have considered the time driven approach, but in our work we have considered the event driven approach. Our work is efficient to a single cluster. In our proposed system we have considered positioning a sink in single cluster to the optimal position. In this we proved using mathematical model for single cluster. Further more than a sink and to multicluster. The mathematical model we proposed can be extended to the multi sink, so that still the energy of the sensors can be reduced over communication and thereby elongating the life of the sensor network. To distributed event and for multi sources also. If we are achieving energy of sensors with low remaining power, another exciting research challenge has to be taken in to account the actual power levels of the sensor nodes during its process. 


\section{REFERENCES}

[1] Loukas Lazos and Radha Poovendran James A. Ritcey "On the Deployment of Heterogeneous Sensor Networks for Detection of Mobile Targets"

[2] Xiaochun Xu and Sartaj Sahni"Approximation Algorithms for Wireless Sensor Deployment"

[3] Mike Chen, Weidong Cui, Victor Wen, Alec Woo "Security and Deployment issues in a Sensor Network".

[4] Urs Bischo., Martin Strohbach, Mike Hazas, and Gerd Kortuem "Constraint-Based Distance Estimation in Ad-Hoc Wireless Sensor Networks".

[5] Xiang Ji, Hongyuan Zha,"Sensor Positioning in Wireless Ad-hoc Sensor Networks Using Multidimensional Scaling

[6] S Archana Bharathidasan, Vijay Anand Sai Ponduru, "Sensor Networks: An Overview"

[7] Dragos Niculescu and Badri Nath fdnicules,"Ad Hoc Positioning System (APS)".

[8] Wint Yi Poe and Jens B. Schmitt disco,"Minimizing the Maximum Time delay in Wireless Sensor Networks by Intelligent Sink Placement “. Technical Report No. 362/07

[9] Zoltan Vincze, Rolland Vida Attila Vidacs, "Deploying Multiple Sinks in Multi-hop Wireless Sensor Networks".

[10] Jingbin Zhang, Ting Yan, and Sang H. Son, "Deployment Strategies for Differentiated Detection in Wireless Sensor Networks".

[11] Jhon Heidemann Nirupama Bulusu, "Using Geospatial Information in Sensor Network".

[12] Wei Li and Christos G. Cassandras," A MinimumPower Wireless Sensor Network Self-Deployment Scheme".

[13] Katayoun Sohrabi, William Merrill, Member, IEEE, Jeremy Elson, Lewis Girod, Fredric Newberg, and William Kaiser, Senior Member, IEEE, "Methods for Scalable Self-Assembly of Ad Hoc Wireless Sensor Networks ". 